\title{
Proliferating cell nuclear antigen and $S$ phase fraction in endometrial stromal sarcoma
}

\author{
J B Schofield, J Mansi, R S Camplejohn, D P Lane, C Fisher
}

\begin{abstract}
Aims: To investigate the value of immunohistochemical staining for the cell cycle protein proliferating cell nuclear antigen (PCNA) and flow cytometric S phase fraction in determining prognosis in endometrial stromal sarcoma, graded according to mitotic count.

Methods: Seventeen endometrial stromal sarcomas from 13 patients treated at the Royal Marsden Hospital were analysed. Serial $5 \mu \mathrm{m}$ sections were cut for haematoxylin and eosin and immunohistochemical staining for PCNA, performed using the murine monoclonal antibody PC10. PCNA positivity was expressed as a percentage of the total number of cells (PCNA index). Flow cytometric analysis was performed on nuclei extracted from paraffin wax sections.

Results: In the five patients who died of disease within five years, PCNA index varied between $<1 \%$ and $60 \%$ (mean $21 \%$ ) and $S$ phase fraction ranged from 11.3 and 20.1 (mean 13.8). Four patients who were apparently cured showed PCNA indices ranging from $<1 \%$ to $5 \%$ (mean $1.75 \%$ ) and $S$ phase fraction ranging from 1.4 to 3.5 (mean 2.3); and three patients alive with disease showed PCNA indices ranging from $1 \%$ to $15 \%$ (mean $8.6 \%$ ) and $S$ phase fraction ranging from 1.4 to 3.5 (mean 2.3). One patient who died from indolent local disease after nine years showed a PCNA of 1 or less and an S phase fraction of 0.9 .

Conclusions: PCNA staining was variable and therefore not a reliable prognostic indicator, but a high PCNA index was only found in those patients dying of disease within five years. A stronger association was seen between $S$ phase fraction and prognosis; this also correlated well with histological grade determined by mitotic count. In individual borderline cases that are between low and high grade categories, these procedures may be useful.
\end{abstract}

Endometrial stromal sarcomas are uncommon as are reports of case series. ${ }^{1-11}$ Endometrial stromal sarcoma has traditionally been divided into histologically high and low grades on the basis of mitotic count $(>10 / 10$ high power fields denoting high grade) ${ }^{2}$; implicit in the definition is a morphological similarity with the cells of normal endometrial stroma. Those sarcomas apparently arising in endometrium which do not display this feature are coming to be regarded as "poorly differentiated endometrial sarcomas" or just "endometrial sarcomas." 3 Counting of mitotic figures is not always easily reproduced and may not be comparable between different cases, ${ }^{12}$ rendering the reliability of the separation of tumours into low and high grades open to speculation. There is a reported correlation between grade and prognosis, supported by several authors; although low grade tumours are normally indolent and locally recurrent over a prolonged period, they may metastasise. It has also been suggested that mitotic count may not be the most useful criterion for predicting biological behaviour in these lesions, and that it is not necessarily directly related to $S$ phase fraction. ${ }^{9}$ We have previously reported clinical data for a group of endometrial stromal sarcomas. ${ }^{11}$ This study correlates these clinical data with results of three methods of assessing cell proliferation: mitotic count; $S$ phase fraction determined by flow cytometry; and immunohistochemical reactivity with PC 10 (an antibody to proliferating cell nuclear antigen). ${ }^{13}$

\section{Methods}

Between 1976 and 199123 patients were treated at the Royal Marsden Hospital with a diagnosis of endometrial stromal sarcoma. Nine cases for which pathological material was not available were excluded from the study. Histological review excluded one case because it was a high grade sarcoma but not of endometrial stromal type. Paraffin wax blocks were available from 17 tumours from the 13 remaining patients.

Serial $5 \mu \mathrm{m}$ sections were cut from representative blocks for haematoxylin and eosin staining and immunohistochemistry, followed by a $50 \mu \mathrm{m}$ section for flow cytometry. Proliferating cell nuclear antigen (PCNA) immunostaining was performed using the method of Hall et al. ${ }^{14}$ In brief, sections were dried on to slides overnight to avoid heating, then incubated overnight with the murine monoclonal antibody PC10, ${ }^{13}$ using a working dilution of 1 in 500, followed by application of an immunoalkaline phosphatase technique with a streptavidin biotin amplification system.

Counting was performed by one observer (JS) using an Olympus BH2 microscope with $\times 40$ objective and a $10 \times 10$ square eyepiece graticule. At least 1000 cells were counted in each case in randomly assorted fields and the percentage of cells showing positive staining (PCNA index) calculated. Counting was 
Endometrial stromal sarcomas: grading, proliferation indices, and follow up

\begin{tabular}{|c|c|c|c|c|c|c|c|}
\hline Case No & Grade & $\begin{array}{l}\text { Mitoses } \\
\text { /10hpf }\end{array}$ & $\begin{array}{l}P C N A+ \\
\%\end{array}$ & $C V$ & $\begin{array}{l}S \text { phase } \\
\text { fraction }\end{array}$ & Follow & $u p$ \\
\hline 1 & Low & 1 & 1 & $4 \cdot 2$ & 1.4 & NSR & \\
\hline 2 & Low & 1 & 1 & $3 \cdot 5$ & $2 \cdot 0$ & NSR & \\
\hline 3 & Low & 3 & 2 & $3 \cdot 1$ & $2 \cdot 2$ & AWD & \\
\hline 4 & Low & 1 & $<1$ & 5.9 & $0 . \overline{9}$ & DOD & $111 \mathrm{~m}$ \\
\hline $4 \dagger$ & Low & $<1$ & 1 & 11 & NA & DOD & $111 \mathrm{~m}$ \\
\hline $4 \dagger$ & Low & $<1$ & $<1$ & 11 & NA & DOD & $111 \mathrm{~m}$ \\
\hline 5 & Low & $<1$ & $<1$ & 11 & NA & NSR & \\
\hline 6 & Low & 5 & 5 & 6.5 & 3.5 & NSR & \\
\hline 7 & Low & 2 & 1 & 11 & NA & AWD & \\
\hline $7 \dagger$ & Low & 6 & 10 & 11 & NA & AWD & \\
\hline $7 \dagger$ & High & 40 & 15 & 8 & $7 \cdot 7$ & AWD & \\
\hline 8 & High & $>10$ & 5 & $6 \cdot 6$ & 11.5 & DOD & $22 \mathrm{~m}$ \\
\hline 9 & High & 25 & 15 & $4 \cdot 7$ & 3.5 & AWD & \\
\hline 10 & High & 20 & 20 & 11 & NA & DOD & $11 \mathrm{~m}$ \\
\hline 11 & High & 30 & 20 & $5 \cdot 3$ & $11 \cdot 3$ & DOD & $? \mathrm{~m}$ \\
\hline 12 & High & 50 & $<1$ & $3 \cdot 6$ & 12.5 & DOD & $12 \mathrm{~m}$ \\
\hline 13 & High & 80 & 60 & $6 \cdot 5$ & $20 \cdot 1$ & DOD & $11 \mathrm{~m}$ \\
\hline $14^{\star}$ & $\mathrm{High}^{\star}$ & 50 & 65 & $8 \cdot 8$ & $26 \cdot 2$ & NA & \\
\hline
\end{tabular}

*reclassified as non-stromal endometrial sarcoma

trecurrence

AWD = alive with disease; $\mathrm{DOD}=$ dead from endometrial stromal sarcomas; $\mathrm{NSR}=$ no signs of recurrence; $\mathrm{NA}=$ not available. repeated for consistency. Mitotic counting was performed on haematoxylin and eosin stained sections; at least 40 fields were counted and the results expressed as number of mitoses per 10 high power fields (HPF). Tumours were allo-

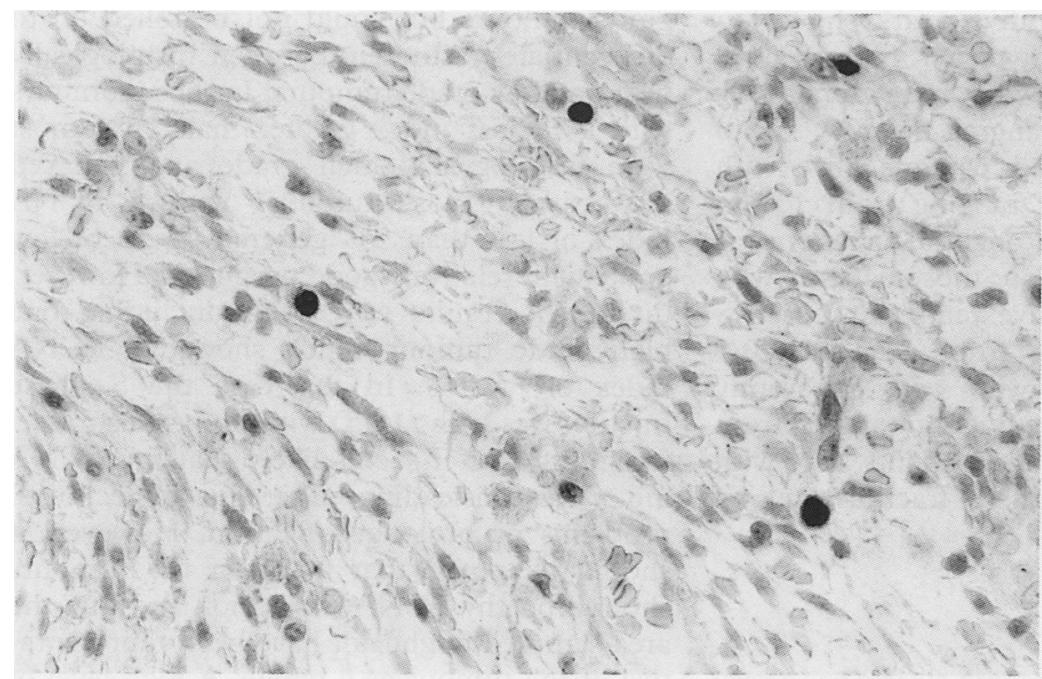

Figure 1 Immunohistochemical localisation of PCNA in low grade endometrial stromal sarcoma; a few cells show positive staining for PC 10.

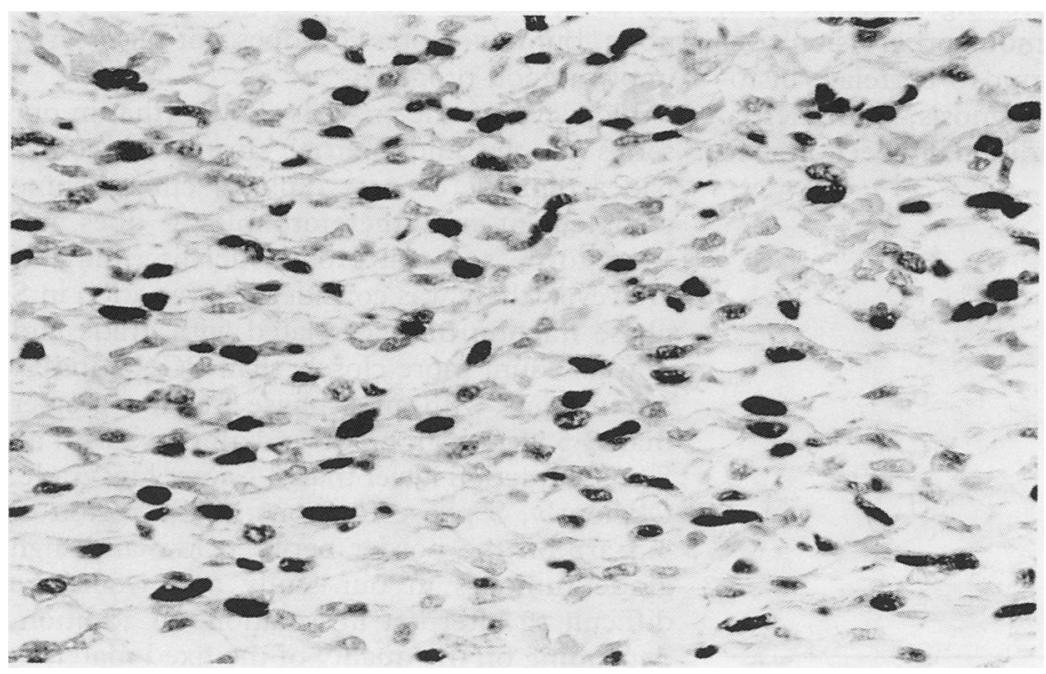

Figure 2 Immunohistochemical localisation of PCNA in high grade endometrial stromal sarcoma; there is extensive nuclear and cytoplasmic staining for PC 10. cated to high or low grade categories on the basis of mitotic activity using the method of Norris and Taylor, ${ }^{2}$ more than 10 mitoses per high power field indicating high grade morphology.

Flow cytometric analysis was performed on nuclei extracted from $50 \mu \mathrm{m}$ paraffin wax sections by treatment with pepsin, as described by Camplejohn et al. ${ }^{15}$ At least 10000 nuclei were scanned to construct each DNA histogram. As all the DNA histograms for the cases of endometrial stromal sarcomas showed a single diploid Gl peak, the fraction of $S$ phase cells was calculated by the method of Baisch $e t$ $a l .{ }^{16}$ For the one non-stromal sarcoma, $S$ phase fraction was estimated by a modification of the Baisch method. ${ }^{15}$ Clinical and follow up information came from hospital case notes.

\section{Results}

The results are shown in the table. There were seven high and 10 low grade tumours. PCNA staining was performed on all cases (figures 1 and 2 ); in many the staining was heterogeneous, but random counting resulted in a considerable range of values $(<1-60 \%)$ which were found to be reproducible on repeated counts. Mean PCNA index in high grade tumours was $19 \cdot 2 \%$ (range $<1-60 \%$ ) and in low grade tumours it was $2.3 \%$ (range < $1-10 \%)$. Five of seven high grade tumours had a PCNA index of $15 \%$ or greater; nine of 10 low grade tumours had a PCNA index of 5\% or less. The one low grade tumour with a higher PCNA index (10\%) was a recurrence (the initial tumour PCNA index was $<1 \%$ ); this patient had a further recurrence which histologically was high grade (PCNA index $15 \%)$. Two high grade tumours had low PCNA indices $(<1 \%$ and $5 \%)$ despite considerably increased mitotic activity and high $S$ phase scores. One of these patients died of aggressive local disease at 22 months and the other died of aggressive local disease and bone metastases at 12 months. PCNA index and its relation to histological grade is shown in fig 3.

Satisfactory flow cytometric data were obtained for only 11 tumours (six high and five low grade) from 11 patients, the remaining six having a coefficient of variation (CV) of greater than 10. None of the tumours was aneuploid, but there was a striking variation in $S$ phase fraction $(0 \cdot 9-20 \cdot 1)$. Mean $S$ phase in high grade tumours was $11 \cdot 1$ (range $3 \cdot 6-20 \cdot 1$ ) and in low grade tumours it was 2.0 (range $0 \cdot 9-3 \cdot 5)$. The excluded "non stromal" endometrial sarcoma had the highest $S$ phase fraction (26.2) and showed $48 \%$ aneuploidy. $S$ phase fraction and its relation to grade is shown in fig 4.

Follow up information was available on all cases; of 13 patients, six had high grade and six had low grade tumours; one further patient was initially classified as low grade (substantiated on review), but a subsequent biopsy specimen showed features of high grade endometrial stromal sarcoma. She was alive 52 months after diagnosis, with persistent disease in the pelvis and lungs following multiagent 
Figure $3 P C N A$ index and histological grade.

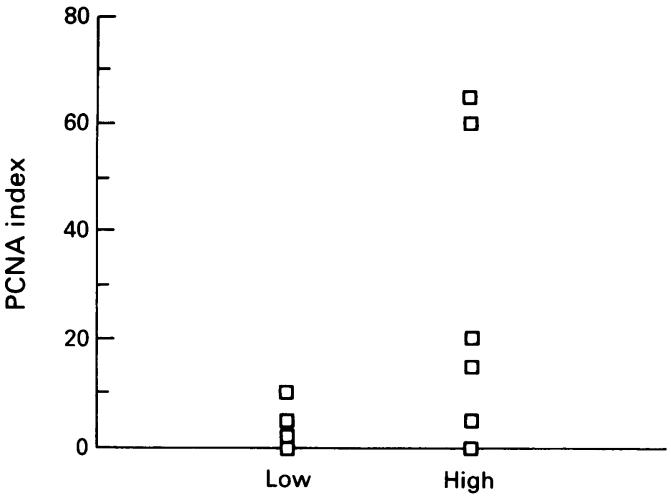

Histological grade

cytotoxic chemotherapy. Five of the remaining six patients with high grade tumours have died (interval to death 11-22 months); one patient was alive with extensive local disease following chemotherapy at the time of writing. Of the other patients with low grade tumours, four were alive without recurrence, one had recurrent local disease, and one died eight years after initial diagnosis with extensive local disease and pulmonary metastases.

The PCNA index showed no clear association with outcome (table); those patients who died of disease within five years showed a PCNA index varying between $<1 \%$ and $60 \%$ (mean 21\%). Patients who were apparently cured showed indices ranging from $<1 \%$ to $5 \%$ (mean $1.75 \%$ ), and patients alive with disease showed indices ranging from $1 \%$ to $15 \%$ (mean $8.6 \%$ ). A stronger association was seen with $S$ phase fraction; those patients who died of disease within five years showed an S phase fraction ranging from 11.3 to $20 \cdot 1$ (mean 13.8). Patients who were apparently cured showed an $S$ phase fraction ranging from 1.4 to 3.5 (mean $2 \cdot 3$ ), and patients alive with disease showed an $S$ phase fraction ranging from 2.5 to $7 \cdot 7$ (mean 4.5 ).

\section{Discussion}

Endometrial stromal sarcoma clearly has a very variable clinical course, some patients dying of disease within months of diagnosis and others having a much more prolonged course with recurrence and slow tumour progression over many years. In an attempt to correlate histological findings with prognosis, endometrial stromal sarcoma has traditionally been graded

Figure $4 S$ phase fraction and histological grade.

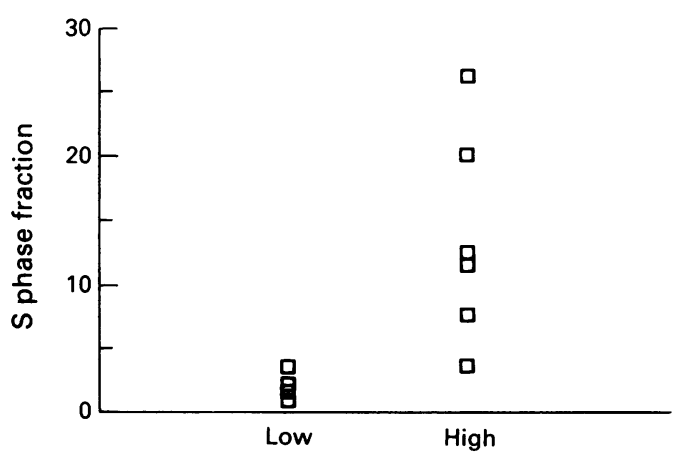

Histological grade on the basis of mitotic activity. ${ }^{2}$ No other histological features have yet been shown to be of value in this regard. It is now becoming increasingly apparent that measurement of mitotic activity is a relatively poor index of cell proliferation. ${ }^{17}$ Alternative approaches to this problem include estimation of cell cycle associated proteins and measurement of $S$ phase fraction determined by flow cytometry. Proliferating cell nuclear antigen (PCNA) is a 36 kilodalton nuclear protein present in the nucleus of proliferating cells which is essential for DNA replication. PC10 is a monoclonal antibody reactive with a particular epitope of PCNA (polymerase $\partial$ accessory protein), which has the advantage of stability following routine histological processing and paraffin wax embedding, permitting investigation of this marker of cell proliferation using archival material. ${ }^{13}$ PCNA has been shown to be increased in amounts in some tumours including non-Hodgkin's lymphoma where a linear correlation has been shown between immunohistochemically confirmed PCNA activity and $S$ phase fraction, ${ }^{14}{ }^{18}$ but there is a less certain correlation in some other tumours. ${ }^{14}$

In our cases of endometrial stromal sarcomas mean PCNA index was higher in the high grade tumours but could not be relied on to differentiate accurately between histologically high and low grade tumours. In those tumours with a PCNA index greater than $15 \%$ most were histologically high grade and behaved aggressively; those tumours with a PCNA index of less than $5 \%$ generally were of low grade with relatively good prognosis. However, discrepant results were seen, including one high grade tumour which showed a PCNA index of $<1 \%$. In this case the $S$ phase fraction was high $(12 \cdot 5)$ with numerous mitotic figures, and it seems likely that the PCNA index was misleading either due to technical problems or prolonged fixation. However, it should be noted that many of the tumours showed patchy PCNA expression and that the area sampled might not have been representative of the tumour as a whole. A high level of PCNA expression, particularly in a tumour showing low grade or borderline histological features, may be useful in defining poor prognosis, but low expression does not necessarily imply a good prognosis.

Flow cytometric analysis showed that in our series all the endometrial stromal sarcomas had a diploid (or near diploid) chromosome complement and therefore ploidy does not seem to be a useful prognostic discriminant. However, there was considerable variation in $S$ phase fraction that correlated with grade and mitotic count more closely than PCNA index. All tumours with an $S$ phase fraction greater than 3.5 were high grade and all those with an $S$ phase fraction lower than 3.5 were low grade. Obviously, $S$ phase fractions in the region of 3.5 are on the border between low and high grade tumours in our study and would be difficult to interpret in isolation. In addition, depending on the quality of the fixed material, flow cytometric data may not be satisfactory (no data were obtained for $35 \%$ of cases in this 
study). However, this success rate can be increased by controlling fixation more carefully (Gillett and Camplejohn, unpublished data). Our findings confirm those of August et al," who showed diploid complement in four cases of "endolymphatic stromal myosis" and one case of endometrial stromal sarcoma. They noted aneuploidy in four further cases referred to as "stromal sarcoma", which, according to their description, might be classified as nonstromal endometrial sarcomas. Indeed, one of the cases originally included in our study was excluded as a "non stromal" tumour; interestingly, this tumour also showed aneuploidy together with a noticeably raised $S$ phase fraction $(26 \cdot 2)$ as well as the highest PCNA index $(65 \%)$. These findings suggest that there is a definite biological difference between the sarcomas of endometrial stromal and those of "non-stromal" type. In the light of this the presence of aneuploidy in endometrial stromal sarcomas should be viewed as a worrying factor and should increase initiate careful histological review of this diagnosis.

The series is too small to know whether the additional information gained by ploidy studies and quantitative measurement of immunohistochemically located PCNA activity adds prognostic information over and above stage and grade. However, it seems likely that in individual cases, particularly those which histologically border between low and high grade, these procedures may be of value.

1 Koss LG, Spiro RH, Brunschwig A. Endometrial stromal sarcoma. Surg Gynecol Obstet 1965;121:531-7.

2 Norris JJ, Taylor HB. Mesenchymal tumours of the uterus.

1. A clinical and pathological study of 53 endometrial stromal tumours. Cancer 1966;19:755-66.

3 Evans HL. Endometrial stromal sarcoma and poorly differ- entiated endometrial sarcoma. Cuncer 1982;50:2170 82

4 Kat\% L, Merino M, Sakamoto H, Schwart\% PE. Endometrial stromal sarcoma: a clinicopathological study with determination of estrogen and progestin receptor levels in three tumours. Gynecol Oncol 1985;26:87-97.

5 George M, Pejovic MH, Kramar A. Uterine sarcomas: Prognostic factors and treatment modalities--a study on 209 patients. Gynecol Oncol 1986;24:58-67.

6 Covens AL, Nisker JA, Chapman WB, Allen HH. Uterine sarcoma: an analysis of 74 cases. Am 7 Obstet Gymiol 1987;156:370-4.

7 Taina E, Maenpaa J, Erkkola R, Ikkala J, Soderström O, Viitanen A. Endometrial stromal sarcoma: A report of nine cases. Gynecol Oncol 1989;32:156-62.

8 De Fusco PA, Gaffey TA, Malkasian GD, Long HJ, Cha SS Endometrial stromal sarcoma: Review of Mayo Clinic experience, 1945-1980. Gynecol Oncol 1989;35:8-14.

9 August CZ, Bauer KD, Lurain J, Murad T. Neoplasms of endometrial stroma: Histopathologic and flow cytometric analysis with clinical correlation. Hum Pathol 1989;20:232-7.

10 Larson B, Silfversward C, Nilsson B, Pettersson F. Endometrial stromal sarcoma of the uterus. A clinical and histopathological study. The radiumhemmet series 1936-1981. Eur f Obstet Gynecol Reprod Biol 1990; 1936-1981.
35:239-49.

11 Mansi JL, Ramachandra S, Wiltshaw E, Fisher C. Endometrial stromal sarcomas. Gynecol Oncol 1990; 36:113-18.

12 Silverberg SG. Reproducibility of the mitosis count in the histological diagnosis of smooth muscle tumours of the uterus. Hum Pathol 1976;4:451-4.

13 Waseem NH, Lane DP. Monoclonal antibody analysis of the proliferating cell nuclear antigen (PCNA); Structural conservation and the detection of a nucleolar form. $f \mathrm{Cell}$ Sci 1990;96:121-9.

14 Hall PA, Levison DA, Woods AL, et al. Proliferating cell nuclear antigen (PCNA) immunolocalisation in paraffin nuclear antigen (PCNA) immunolocalisation in paraffin
sections: an index of cell proliferation with evidence of sections: an index of cell proliferation with evidence of
deregulated expression in some neoplasms. $\mathcal{F}$ Pathol deregulated expressi

15 Camplejohn RS, Macartney JC, Morris RW. Measurement of $\mathrm{S}$-phase fractions in lymphoid tissue comparing fresh versus paraffin embedded tissue and 4', 6'-diamidino-2 phenylindole dihydrochloride versus propidium iodide staining. Cytometry 1990;10:410-16.

16 Baisch H, Gohde W, Linden WA. Analysis of PCP-data to determine the fraction of cells in the various phases of the cell cycle. Radiat Environ Biophys 1975;12:31-9.

17 Hall PA, Levison D. Assessment of cell proliferation in histological materials. f Clin Pathol 1990;43:184-92.

18 Woods AL, Hall PH, Shepherd NA, et al. The assessment of proliferating cell nuclear antigen (PCNA) immunostaining in primary gastrointestinal lymphoma and its relationship to histological grade, $S+G_{2}+M$ fraction (flow cytometric analysis) and prognosis. Histopathlogy 1991;19:21-7. 